\title{
High resolution temporal network analysis to understand and improve collaborative learning
}

\author{
Mohammed Saqr ${ }^{\dagger}$ \\ School of Computing \\ University of Eastern Finland \\ Joensuu, Finland \\ Mohammed.saqr@uef.fi
}

\author{
Jalal Nouri \\ Department Of computer and \\ systems science \\ Stockholm University \\ Stockholm, Sweden \\ jalal@dsv.su.se
}

\begin{abstract}
There has been significant efforts in studying collaborative and social learning using aggregate networks. Such efforts have demonstrated the worth of the approach by providing insights about the interactions, student and teacher roles, and predictability of performance. However, using an aggregated network discounts the fine resolution of temporal interactions. By doing so, we might overlook the regularities/irregularities of students' interactions, the process of learning regulation, and how and when different actors influence each other. Thus, compressing a complex temporal process such as learning may be oversimplifying and reductionist. Through a temporal network analysis of 54 students interactions (in total 3134 interactions) in an online medical education course, this study contributes with a methodological approach to building, visualizing and quantitatively analyzing temporal networks, that could help educational practitioners understand important temporal aspects of collaborative learning that might need attention and action. Furthermore, the analysis conducted emphasize the importance of considering the time characteristics of the data that should be used when attempting to, for instance, implement early predictions of performance and early detection of students and groups that need support and attention.
\end{abstract}

\section{CCS CONCEPTS}

Applied computing $\rightarrow$ Education $\rightarrow$ Collaborative learning

\section{KEYWORDS}

Learning analytics, Social network analysis, Temporal networks, Temporarily, Collaborative learning, Problem-based learning, Medical education

Permission to make digital or hard copies of part or all of this work for personal or classroom use is granted without fee provided that copies are not made or distributed for profit or commercial advantage and that copies bear this notice and the full citation on the first page. Copyrights for third-party components of this work must be honored. For all other uses, contact the Owner/Author. LAK '20, March 23-27, 2020, Frankfurt, Germany $\odot 2020$ Copyright is held by the owner/author(s).

ACM ISBN 978-1-4503-7712-6/20/03.

https://doi.org/10.1145/3375462.3375501

\section{ACM Reference format:}

Mohammed Saqr, Jalal Nouri. 2020. High resolution temporal network analysis to understand and improve collaborative learning. In Proceedings of the $10^{\text {th }}$ International Conference on Learning Analytics \& Knowledge (LAK'20). ACM, New York, NY, USA, 6 pages.

\section{Introduction}

Today massive amounts of data are generated in blended and online learning environments. For a number of years now, researchers in the field of technology-enhanced learning, and learning analytics in particular, have used such data for data-driven analysis "for purposes of understanding and optimizing learning and the environments in which it occurs" [24]. However, despite that most of the generated or collected data is time-stamped, temporal analysis that consider when different learning processes and interactions take place has been understudied $[4,7,23]$. And by not taking temporal aspects into account we overlook the fact that learning involves processes that occurs over time $[7,16,23,25]$; and that the nature and quality of the learning processes is dependent on when, for instance, interactions with learning material, peers or teachers is taking place. The tendency so far, as stated by [25], has been to "compress, if not totally ignore, the temporal dimension by using "code and count" methods that aggregate over time and operating in "snapshot" mode-showing users a current picture of the data-which can overlook or misrepresent patterns that change over time" [25].

Thus, the main limitations of current approaches are that they are not providing information to education practitioners that is fully usable due to that time is overlooked. As argued by [13,25] the methodological approaches to "compare" and "correlate" aggregated variables fails to fully utilize the temporal information embedded in the data [13,25]. Such information reduction results in both a limitation in validity of conclusions (ibid.) and in our ability to understand the learning processes, detect students who may need support, and most importantly understand when we should intervene. Collaborative learning in online and blended learning environments is one of the areas that could benefit if the temporal dimension of learning is accounted for; $[10,16]$ emphasize this by pointing out that it is essential to acknowledge that collaborative processes unfold over time and that progress in collaboration is critical for learning success $[10,16]$, which underscore the importance of considering when and 
how collaborative learning contribute to knowledge construction. In the studies of $[10,16,17]$, which are among the very few studies, temporal aspects of self-regulation in collaborative settings where investigated, contributing to the empirical evidence demonstrating the importance of incorporating temporal characteristics $[10,16,17]$. None of the mentioned studies, however, applied Social Network Analysis (SNA) which has become one of the most commonly applied methods in learning analytics research [14], and that has been shown to be useful for the analysis of collaborative learning [23]. In the context of online and blended learning environments, we are only aware of one study by [27] that employed temporal networks to model the relational events in a large MOOC to predict future success and course drop-outs [27]. Another study by [14] who employed temporal analysis to trace student ideas and determine their impact on communal discourse [14].

\subsection{Network Analysis}

Interactions among learners can be represented as a network, where each actor is represented as a node and the interaction as an edge. Results are usually compiled as an aggregated network of all of the interactions at a certain point in time, typically end of course $[5,8,27]$. Significant progress has been achieved by providing educators with tools and methods to visualize, quantify and mine relations and interactions, map collaborators roles, rank important collaborators and forecast students' future behavior or performance. Methods include quantitative network analysis, generative network models and visualization $[5,11,12,21]$. This study extends current approaches by including the time dimension. Temporal networks or time-varying graphs represent interactions that can be modelled using time as an ordered sequence of edges. There are two common classes of temporal networks (sometimes overlapping): a contact sequence network in which the duration of contact is so trivial such as email networks and instant messages. In such networks, the contact is instantaneous and the duration can be negligible. The second type is interval networks, where interactions occur over a duration of time such as proximity networks $[8,18]$. In interval networks, the duration of contact or interaction is considerable. In this study, we represent interactions in the context of online collaborative learning as a time interval temporal network, building on the methodology described by [27]

This study builds upon previous work by on the one hand continuing the line of work that studies temporal aspects of collaborative learning in blended and online environments, and on the other hand, by further investigating the usefulness of building, visualizing and quantitatively analyzing temporal networks as a lens to gain insights into how the dynamics of students' collaborative interactions unfold, progress and influence productive co-construction of knowledge. The study also demonstrates how quantitative temporal indicators could inform educators about the status of collaborators and help build timely predictive models aiming at supporting educational practitioners to provide proactive actions. We try to explore the value of temporal high resolution network analysis in monitoring interactions, early prediction of performance and whether temporal network indicators offer a better alternative to statics measures?

\section{Methodology}

Context and data collection: The study was performed in the body systems course in Qassim University. The university implements a problem based curriculum, the problem-based process occurs mostly online. The approach relies on three elements: An on open ended problems as a trigger for interactions, small groups and a moderating tutor. The PBL process is structured into sequential steps, each week, students are offered a clinical case scenario or a real-life open ended questions, students should start the process by clarifying the terms, identify the problem, brainstorm, formulate a hypothesis, and lastly formulate the learning objectives. Thereafter, students should share, debate and refine their understanding of the problem. On the last two days of the week, students should conclude, synthesize, reflect on the process and receive feedback from peers and tutors. The interactions occur on the Moodle learning management system forum module. Each PBL group had a separate online forum thread for each week, and the forum is timed, so it is started on the Monday, and receives no posts after Friday. As the curriculum is PBL based, the PBL is the central element of organizing the course, the objectives of the PBL constitute the week's theme of the lectures and practical. The final course exam evaluates the knowledge, attitude, and skills based on the PBL objectives. Performance in this study was measured using three measures: the assessment (students in-class and online engagement as evaluated by the instructors), final exam which is the score of the end of course exams (theory, practice and skills) and the total which is the final grade (sum of all exams and evaluations). The students were ranked according to final grade into high and low achievers (top and bottom 50\%). The interactions data were extracted from Moodle. The retrieved data included the users IDs, enrollments, grades, interactions and the metadata associated with each interaction (post author, group, thread ID, parent ID, timestamp, subject, content and replies).

\subsection{Analysis}

To build a temporal networks (interval network in our case), four elements are usually required: a source, a target, an onset and a duration or offset for each edge. In this study, edges were constructed from each post by considering the author of the post as the source, the replied-to-user as the target, the timestamp of the post was the onset, the duration was the interval between the onset and the timestamp of the latest activity of the thread, similar to the approach of [27]. An aggregate static network was also constructed for comparison of indices calculated from both networks.

The data were analyzed using $\mathrm{R}$ programming language [20]. The temporal network analysis was performed with Network Dynamic Temporal Visualizations library (ndtv) [1] and tSNA package [3]. Correlations were performed using Spearman correlation test. Linear regression with a 10 -fold cross-validation was performed to predict performance. The model diagnostics reported are the Mean Squared Error (MSE), Root Mean Squared Error(RMSE) and the Mean Absolute Error (MAE). Entropy was used to measure the regularity/irregularity of students' activity and was calculated using the tsfeatures package [9].

Quantitative network statistics: Dynamic network statistics are calculated in a different way than their corresponding static ones by 
considering a time scale for aggregating. For this study we selected the time scale of one day to have a high resolution view of the network dynamics. Two types of network statistics were calculated, network and actor level statistics. The calculated network level parameters were selected to reflect network interactivity, cohesion, reciprocity and structure of ties. All dynamic network indices were computed using the tSNA package [3]. Dynamic density was calculated as the fraction of active observed edges for every day. Dynamic mutuality represented the number of daily reciprocated active edges. Dynamic mean degree, is the total number of active edges at each day divided by the number of users. Simmelian ties are the number of subgraphs of size three at the day level. Density, mutuality and Simmelian ties are measures of network cohesion while mean degree is an indication of activity [2,11]. On the actors level, the dynamic indegree/outdegree centrality were calculated as the active inbound/outbound interactions during each day. Two methods were used to calculate the dynamic degree: duration of activity and count of interactions during each day. Dynamic Eigen centrality, a measure that emphasizes the connectedness and importance of neighbors, was calculated as the monetary eigen centrality of each day. Dynamic betweenness flow centrality (flow) was selected over traditional betweenness centrality to overcome the shortcomings of betweenness that counts only the shortest paths that go through an actor. Dynamic Flow centrality considers all paths that go through the node, by doing so it accounts for all times that an actor connects others, or mediates information exchange. Dynamic closeness centrality was calculated but since the results were undefined for most of the days due to isolates and disconnected networks, it was excluded $[3,15,18]$. Lastly, for comparison, the static indegree, outdegree, eigen and flow centralities were calculated for the static network. To measure regularity/ irregularity, we used the entropy score. Entropy is expected to be high for irregular process and low for regular or predictable dynamic processes [9].

\section{Results}

The study included 54 students attending a body systems course. The number of interactions was 3134, 2768 (91.8\%) were among the students, $9(0.3 \%)$ were among the teachers and the $7.9 \%$ mixed interaction. 28 interactions in 3 threads were after the course ended and so were excluded. The mean density of interactions within the groups was 0.78 (ranged from 0.51 to 0.93 ). In contrast to static networks statistics which are usually expressed as a single summary value, temporal networks enable continuous evaluation of graph properties. We plotted the graph properties at the resolution of a single day (weekends removed) in Figure 1. The density, mean degree, and reciprocity followed a cyclic burst pattern that reflected the course design. However, there was a trough period in the last two or three days of each week representing a time of slow or no activity. The trough got wider as the course advanced. This temporal pattern reflected students' engagement with the early phases of the PBL process and disengagement with the late phase, the disengagement was less marked early in the course, which reflects an obvious gap in the online PBL process. By being less active in the late phase, students were spending limited time on collaboration, reflection, synthesis and feedback, which are integral phases of the process of
PBL. The simmelian ties graph, shows the same cyclic pattern, however, the formation of simmelian ties peaked early in the course and slowed down later.

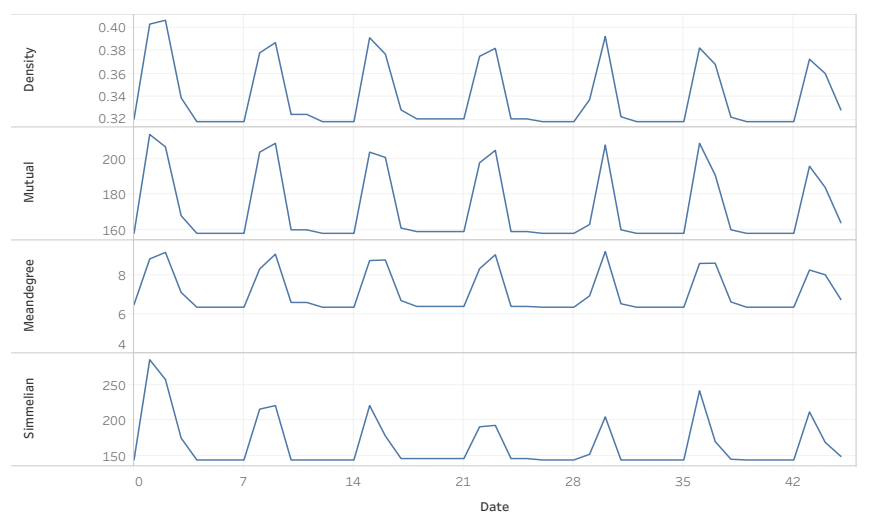

Figure 1 a plot of the daily dynamic density, mutuality, mean degree and simmelian ties across the course.

\subsection{Monitoring interactions}

The value of real-time monitoring can also be seen by looking at Figure 2, a graph of a group in the first week of the studied course, the group is composed of 10 students and a tutor (node 40). The dynamic network graph shows that students were engaged early during the week and started to disengage on Wednesday, the last two days show a completely disconnected group with no activity. The aggregate network (top raw) at the third, fourth, and fifth day points to a seemingly active group, while, in fact 5 students were disengaged on the third day, and all students were disengaged on the 4th and 5th day. Such fine grained insights are required to identify gaps in the process of learning. This is a stark example of why temporality is a necessary lens to understand learning processes and course design.

Figure 3 shows the types of actors mixing as plotted against time, high-to-high achievers interactions (blue) are engaged consistently earlier in mutual interactions than low-to-low achievers (green). As such, the interactions are usually driven and initiated by the high achievers. In fact, they precede all other types of interactions in the course. The consistent timely and early engagement of higher achievers could support the design of predictive models. Tutor-tohigh and low students are almost similar and synchronous with the students' cyclic pattern. One would expect the tutor to interact and scaffold the low achievers more. The time of tutor interactions may be expected to differ than students' by showing activities outside the time bounds of students interactions. For example, earlier to stimulate interactions, later to stimulate a disengaged group, or to help students reflect and synthesize during the late phase that is poorly implemented. Lastly, the low achievers (yellow line) were more likely to interact with the tutors than the high achievers (light 
blue).

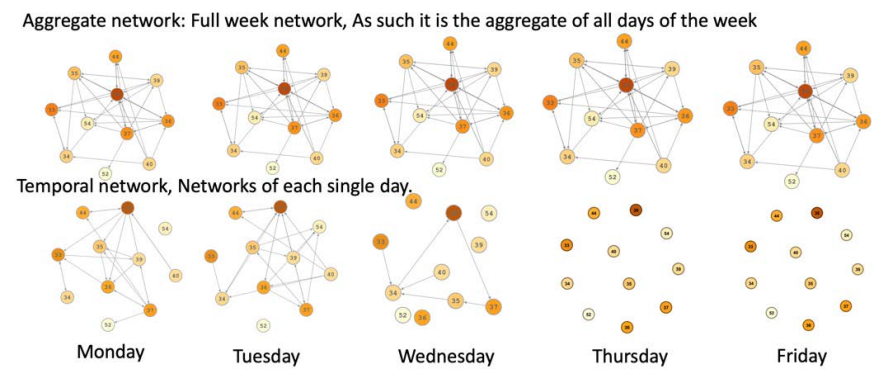

Figure 2 a comparison of an aggregate network graph compared to its corresponding dynamic network graph. The static aggregate network in the top raw is constant, while the temporal network changes on daily basis showing interactivity at every day.

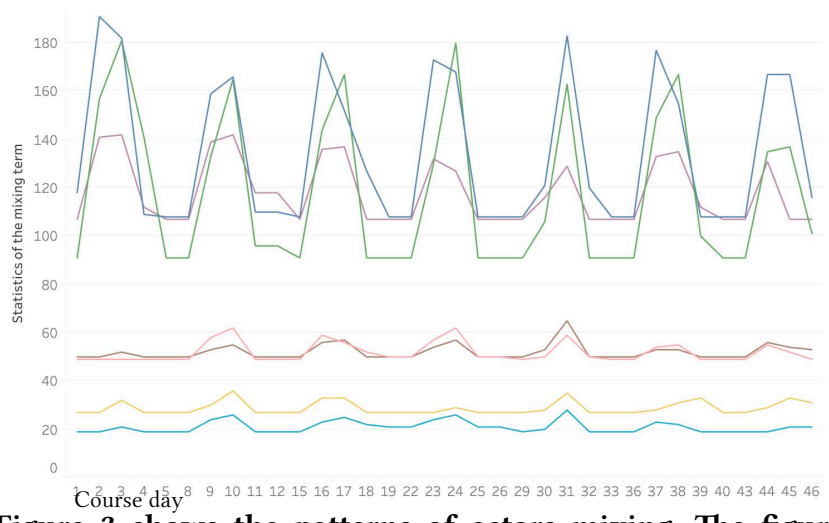

Figure 3 shows the patterns of actors mixing. The figure shows how each actor time-line interact with each other.

\subsection{Early prediction of performance.}

To test if temporal networks with high resolution may improve the opportunities of early predicting performance, we performed a Spearman's correlation test with dynamic centrality measures calculated on a daily basis (except for weekends). Each circle in figure 4 represents a significant positive correlation between a centrality measure and the three performance measures. The results show that daily centrality measures were correlated with performance since early in the course, which may be beneficial in forecasting future performance. The correlation was significant, higher and more consistent on the second and third days of the week. Furthermore, the correlation was higher on the second week and extended to all days of the second and most of the third week. Indegree centrality was almost insignificant on first day of the week as it took some time to receive a reply. The correlation between centrality measures and continuous assessment was usually higher than other performance measures, a sign that temporal network measures are good indicators of engagement with course activity. In summary, the temporality of correlation shows that high achievers are both active early on both week and course levels. The correlation peeks around mid-week, and around mid-course. A researcher wishing to design a predictive model, may be able to understand which dates are important, which dates to aggregate, which to exclude (noise) and which portion of the early data that produce the best results. We will test this further in the next section.
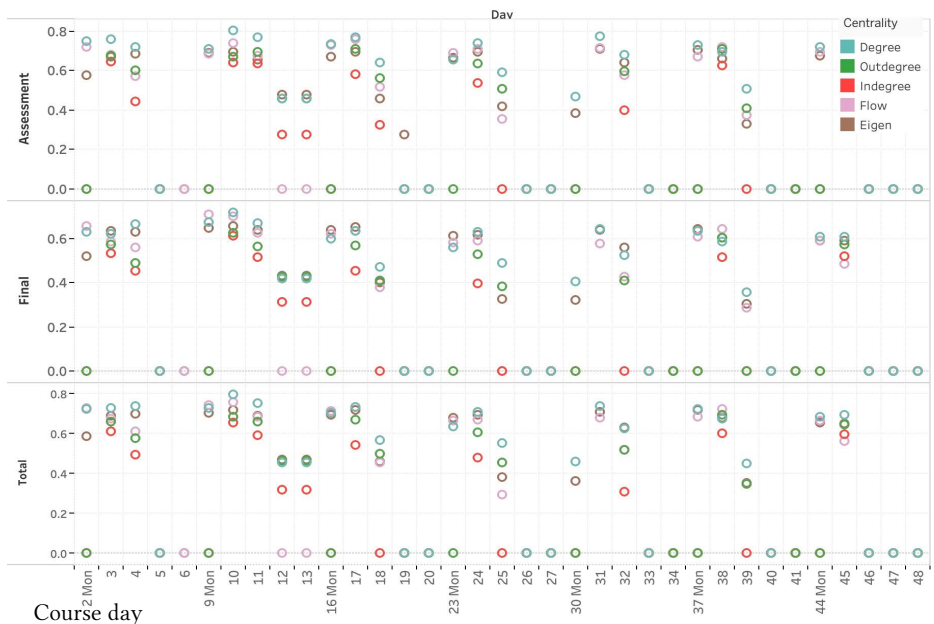

Figure 4 shows correlation among dynamic centrality scores and performance measures (If a correlation is significant it is represented as a circle on the vertical access)

\subsection{Predicting performance}

Given the correlation and visual analysis, the identification of temporality patterns, and informed by the course design that relies on weekly tasks, we hypothesized that a weekly predictive algorithm on the second day of the week may be a rational choice. As shown in Table 1, using the 4 dynamic centrality measures (non-aggregated with previous days), we compare 7 linear regression algorithms, constructed with the 2nd,9th, 16th, 23rd, 30th, 37th and 44th days of the course. The results showed that linear regression performed on the second day of the course had an R2 of 0.456, MAE of 3.8, and RMSE of 4.6, the results are slightly lower than the model calculated with static centralities by the end of the course. A reasonable result given the value of such results early in the course. As was noted in the correlation tests, the 9th day model offered the most accurate model among the other days and verified our hypothesis. The model created with aggregate dynamic centralities (sum) proved to be the best performing model having the highest R2 (0.54) and the lowest, MAE, RMSE and MSE. Meanwhile entropy proved to be the worst with R2 of only 0.05 . It may be also noted that the temporality pattern of students activities was also reflected in the values of R2 of the predictive models. The early models showed higher predictive power and lower error rates, which peaked in the second weak, and then declined towards the end of the course (except for the pre-last week).Furthermore, the correlation between flow and Eigen centralities aggregated over the course time was markedly higher than their corresponding static centrality measures. Entropy of the centrality measures was negatively and significantly correlated with all performance measures. Meaning that irregularity was a negative predictor of performance. However, the magnitude of correlation was low to moderate and less than other centrality measures. 
Table 1. Comparison of predictive linear regression algorithms

\begin{tabular}{|l|l|l|l|l|}
\hline Algorithm & MSE & RMSE & MAE & R2 \\
\hline 2rd day & 21.19 & 4.60 & 3.77 & 0.46 \\
\hline 9th day & 20.79 & 4.56 & 3.77 & 0.47 \\
\hline 16th day & 23.45 & 4.84 & 4.15 & 0.40 \\
\hline 23th day & 27.21 & 5.22 & 4.20 & 0.30 \\
\hline 30st day & 27.97 & 5.29 & 4.45 & 0.28 \\
\hline 37th day & 23.32 & 4.83 & 4.20 & 0.40 \\
\hline 44th day & 30.29 & 5.50 & 4.81 & 0.22 \\
\hline Static & 20.52 & 4.53 & 3.76 & 0.47 \\
\hline Dynamic centralities & 18.07 & 4.25 & 3.61 & 0.54 \\
\hline Entropy & 37.03 & 6.09 & 4.66 & 0.05 \\
\hline
\end{tabular}

\subsection{Does aggregate dynamic centralities offer a better alternative?}

There are different ways to construct networks, weight the edges and aggregate centrality measures in dynamic networks. In this study, we wished to examine the differences between dynamic centrality measures and static ones in terms of correlation with performance. We used the aggregate and entropy of dynamic centrality measures, the former to represent the sum of effort and the latter to represent the regularity. The results detailed in Table 2 showed that static local centrality measures (indegree and outdegree) were more correlated with performance than aggregate dynamic centrality measures. The correlation was also higher than aggregated dynamic centralities weighted by duration or frequency of interactions. These results although needs to be confirmed in further studies - elicit a need to further test and validate alternative aggregation methods for centrality measures.

Table 1. Correlation between static/dynamic centrality measures and performance

\begin{tabular}{|c|c|c|c|c|c|c|}
\hline Outdegree Centrality & \multicolumn{2}{|l|}{ Assessment } & \multicolumn{2}{l|}{ Final } & \multicolumn{2}{l|}{ Total } \\
\cline { 2 - 7 } & $\mathrm{r}$ & $\mathrm{p}$ & $\mathrm{r}$ & $\mathrm{p}$ & $\mathrm{r}$ & $\mathrm{p}$ \\
\hline Static & 0.79 & 0.00 & 0.65 & 0.00 & 0.74 & 0.00 \\
\hline $\begin{array}{l}\text { Dynamic } \\
\text { Duration }\end{array}$ & 0.62 & 0.00 & 0.47 & 0.00 & 0.56 & 0.00 \\
\hline $\begin{array}{l}\text { Dynamic } \\
\text { Count }\end{array}$ & 0.63 & 0.00 & 0.46 & 0.00 & 0.56 & 0.00 \\
\hline Entropy & -0.36 & 0.01 & -0.34 & 0.01 & -0.36 & 0.01 \\
\hline Indegree & & & & & \\
\hline Static & 0.76 & 0.00 & 0.60 & 0.00 & 0.71 & 0.00 \\
\hline $\begin{array}{l}\text { Dynamic } \\
\text { Duration }\end{array}$ & 0.67 & 0.00 & 0.53 & 0.00 & 0.63 & 0.00 \\
\hline $\begin{array}{l}\text { Dynamic } \\
\text { Count }\end{array}$ & 0.68 & 0.00 & 0.52 & 0.00 & 0.62 & 0.00 \\
\hline Entropy & -0.57 & 0.00 & -0.50 & 0.00 & -0.55 & 0.00 \\
\hline Flow centrality & & & & & & \\
\hline Dynamic & 0.82 & 0.00 & 0.68 & 0.00 & 0.78 & 0.00 \\
\hline Static & 0.65 & 0.00 & 0.49 & 0.00 & 0.58 & 0.00 \\
\hline Entropy & -0.47 & 0.00 & -0.55 & 0.00 & -0.55 & 0.00 \\
\hline Eigen Centrality & & & & & & \\
\hline Dynamic & 0.76 & 0.00 & 0.67 & 0.00 & 0.74 & 0.00 \\
\hline Static & 0.57 & 0.00 & 0.56 & 0.00 & 0.60 & 0.00 \\
\hline Entropy & -0.22 & 0.13 & -0.17 & 0.24 & -0.20 & 0.00 \\
\hline
\end{tabular}

\section{Discussion}

This study took as departure point to investigate temporal aspects of collaborative learning in online environments by specifically scrutinizing the usefulness of applying temporal network analysis to gain insights into how the dynamics of students collaborative interactions influence productive co-construction of knowledge, and how the incorporation of temporal features can inform predictive models aiming at supporting educational practitioners to take timely actions.

In contrast to static networks statistics which are usually expressed as a single summary value, the employment of temporal networks in this study enabled us to continuously monitor and visualize dynamic graph properties. The visualization of the dynamic graph properties supported the identification of a repeating temporal patterns showing that students engage in collaborative learning the first days of the weeks and disengage in the later days of the weeks. This problem was not visible using a traditional aggregate network. Thus through the analysis, it became clear that students skipped an essential part of the process of PBL in which students have to develop their metacognitive strategies and get/give feedback for peers and tutors. This finding support the potentials of using visual temporal analysis of learning processes as also shown in the context of understanding assessment $[19,22]$. Furthermore, through a correlation analysis we could conclude that the early week engagement in collaborative interactions as represented by the selected network parameters significantly correlated with students' performance. High achievers engaged consistently earlier in mutual interactions than low achievers, and the interactions were usually driven and initiated by the high achievers. Informed by the visual analysis and the correlation tests that highlighted the temporal engagement pattern in the course, predictive models were developed to forecast performance. The results of the predictive modelling showed that a model created with aggregate dynamic centralities (based on temporal data) representing collaborative engagement during the 9th day of the course, was the best performing and accurate model for predicting performance among other days. These findings have a couple of implications.

One of the implications is that the methodological approach with temporal network analysis can help educational practitioners to understand important temporal aspects of collaborative learning and course design, that might need attention and action. In the case of the course studied, the findings could inform a redesign of the course considering that students repeatedly disengage in collaborative learning in the last days of every week during the course, despite the fact that the course was designed with the aim of including collaborative work all the days of the week. The analysis also could inform the teacher when to intervene, which is an additional value.

Another implication of the temporal network analysis is related to early predictions of performance. We argue that this study contribute to the ongoing discussions about the limitations of current predictive approaches within the field of learning analytics. It has for instance been proposed that produced predictive models shows limitations in generalizability and transferability [6,12,26]. Through temporal network analysis and the incorporation of temporal features, we draw 
three important conclusions. Firstly, this study demonstrate that we can produce predictive models (based on dynamic temporal properties) that outperform traditional static network analysis without the inclusion of the temporal dimension. Secondly, the findings show that temporal network analysis can give precise, finegrained and data-driven guidance to which particular early data points we should use when attempting to produce early predictions of course performance. In the case of the course studied, data representing collaborative work in the beginning of the week, and more specifically, the 9th day of the course, showed to be the basis for the best predictive models. Thirdly, the findings also corroborate [6] study that empirically demonstrated the importance of understanding the course and disciplinary context as an essential step when developing and interpreting predictive models of academic success and attrition [6]. This study builds on [6], who showed that the variation in student characteristics and available trace data across courses limit the generalizability of predictive models, by demonstrating that level of variability associated with predicting academic success is also dependent on temporal features and structures of courses, which further emphasizes the challenges associated with and the limitations of general and transferable predictive models.

This study has just scratched the surface of temporal network analysis in education, there are many unanswered questions and opportunities for further research. Future studies could explore other types of interactions such as temporal interactions between students and resources, interactions among different learning process and temporal discourse analytics. Similarly, research could test alternative edge construction, aggregation methods, or time scales. Further, incorporating temporal network analysis with discourse analysis or multimodal learning analytics may be an interesting area of inquiry.

\section{REFERENCES}

[1] Skye Bender-deMoll. 2018. ndtv: Network Dynamic Temporal Visualizations. Retrieved from https://cran.r-project.org/package=ndtv

[2] Per Block. 2015. Reciprocity, Transitivity, and the Mysterious. Social Networks 40, October: 163-173.

[3] Carter T Butts. 2010. sna: Tools for Social Network Analysis. R package version 2.2-0.

[4] Bodong Chen, Alyssa F Wise, Simon Knight, and Britte Haugan Cheng. 2016. Putting temporal analytics into practice: the 5th international workshop on temporality in learning data. In Proceedings of the sixth international conference on learning analytics \& knowledge, 488-489.

[5] Marielle Dado and Daniel Bodemer. 2017. A review of methodological applications of social network analysis in computer-supported collaborative learning. Educational Research Review 22: 159-180. https://doi.org/10.1016/j.edurev.2017.08.005

[6] Dragan Ga, Shane Dawson, Tim Rogers, Danijela Gasevic, Dragan Gasevic, Shane Dawson, Tim Rogers, Danijela Gasevic, Dragan Ga, Shane Dawson, Tim Rogers, and Danijela Gasevic. 2016. Internet and Higher Education Learning analytics should not promote one size fi ts all: The effects of instructional conditions in predicting academic success. Internet and Higher Education 28: 68-84. https://doi.org/10.1016/j.iheduc.2015.10.002

[7] Iassen Halatchliyski, Tobias Hecking, Tilman Göhnert, and H. Ulrich Hoppe. 2013. Analyzing the Flow of Ideas and Profiles of Contributors in an Open Learning Community. Proceedings of the Third International Conference on Learning Analytics and Knowledge - LAK '13 1, 2: 66-74. https://doi.org/10.1145/2460296.2460311

[8] Petter Holme and Jari Saramäki. 2012. Temporal networks. Physics Reports 519, 3: 97-125. https://doi.org/10.1016/j.physrep.2012.03.001

[9] Rob Hyndman, Yanfei Kang, Pablo Montero-Manso, Thiyanga Talagala, Earo Wang, Yangzhuoran Yang, and Mitchell O'Hara-Wild. 2019. tsfeatures: Time Series Feature Extraction. Retrieved from https://cran.rproject.org/package=tsfeatures

[10] Amy M Johnson, Roger Azevedo, and Sidney K D'Mello. 2011. The Temporal and Dynamic Nature of Self-Regulatory Processes During Independent and Externally Assisted Hypermedia Learning. Cognition and Instruction 29, 4: 471-504. https://doi.org/10.1080/07370008.2011.610244

[11] Srećko Joksimović. 2017. An analytics-based approach to the study of learning networks in digital education settings. Retrieved from https://www.era.lib.ed.ac.uk/bitstream/handle/1842/25819/Joksimovic2017. pdf? sequence $=1$

[12] Srećko Joksimović, Areti Manataki, Dragan Gašević, Shane Dawson, Vitomir Kovanović, and Inés Friss de Kereki. 2016. Translating network position into performance. In Proceedings of the Sixth International Conference on Learning Analytics \& Knowledge - LAK '16, 314-323. https://doi.org/10.1145/2883851.2883928

[13] Manu Kapur, John Voiklis, and Charles K. Kinzer. 2008. Sensitivities to early exchange in synchronous computer-supported collaborative learning (CSCL) groups. Computers \& Education 51, 1: 54-66. https://doi.org/10.1016/j.compedu.2007.04.007

[14] A V Y Lee and S C Tan. 2017. Temporal analytics with discourse analysis: Tracing ideas and impact on communal discourse. In ACM International Conference Proceeding $\quad$ Series, $120-127$. https://doi.org/10.1145/3027385.3027386

[15] Hao Liao, Manuel Sebastian Mariani, Matúš Medo, Yi Cheng Zhang, and Ming Yang Zhou. 2017. Ranking in evolving complex networks. Physics Reports 689: 1-54. https://doi.org/10.1016/j.physrep.2017.05.001

[16] Jonna Malmberg, Sanna Järvelä, and Hanna Järvenoja. 2017. Capturing temporal and sequential patterns of self-, co-, and socially shared regulation in the context of collaborative learning. Contemporary Educational Psychology 49: 160-174. https://doi.org/10.1016/j.cedpsych.2017.01.009

[17] Inge Molenaar and Ming Ming Chiu. 2014. Dissecting sequences of regulation and cognition: statistical discourse analysis of primary school children's collaborative learning. Metacognition and Learning 9, 2: 137-160. https://doi.org/10.1007/s11409-013-9105-8

[18] Vincenzo Nicosia, John Tang, Cecilia Mascolo, Mirco Musolesi, Giovanni Russo, and Vito Latora. 2013. Graph metrics for temporal networks. In Temporal networks. Springer, 15-40.

[19] Zacharoula Papamitsiou and Anastasios A Economides. 2015. Temporal learning analytics visualizations for increasing awareness during assessment. International Journal of Educational Technology in Higher Education 12, 3: 129-147.

[20] R Core Team. 2018. R: A Language and Environment for Statistical Computing. Retrieved from https://www.r-project.org

[21] Mohammed Saqr and Ahmad Alamro. 2019. The role of social network analysis as a learning analytics tool in online problem based learning. BMC Medical Education 19, 1: 1-11. https://doi.org/10.1186/s12909-019-1599-6

[22] Mohammed Saqr, Uno Fors, and Jalal Nouri. 2018. Using social network analysis to understand online Problem-Based Learning and predict performance. PloS one 13, 9: e0203590. https://doi.org/10.1371/journal.pone.0203590

[23] Mohammed Saqr, Jalal Nouri, Uno Fors, Jalal Nouri, and Uno Fors. 2019 Time to focus on the temporal dimension of learning: A learning analytics study of the temporal patterns of students' interactions and self-regulation. International Journal of Technology Enhanced Learning 11, 4: 398. https://doi.org/10.1504/ijtel.2019.10020597

[24] George Siemens. 2013. Learning Analytics: The Emergence of a Discipline. American Behavioral Scientist 57, 10: 1380-1400. https://doi.org/10.1177/0002764213498851

[25] Bodong Chen. Simon Knight, Alyssa Friend Wise. 2017. Time for change: Why Learning analytics needs temporal analysis. Journal of Learning Analytics 4, 3: 7-17. https://doi.org/http://dx.doi.org/10.18608/jla.2017.43.2

[26] Olga Viberg, Mathias Hatakka, Olof Bälter, and Anna Mavroudi. 2018. The current landscape of learning analytics in higher education. Computers in Human Behavior 89, October 2017: 98-110. https://doi.org/10.1016/j.chb.2018.07.027

[27] Duy Vu, Philippa Pattison, and Garry Robins. 2015. Relational event models for social learning in MOOCs. Social Networks 43: 121-135. https://doi.org/10.1016/j.socnet.2015.05.001 